\title{
Caudal Regression and Tracheoesophageal Malformation Induced by Adriamycin: A Novel Chick Model of VATER Association
}

\author{
YOKO NAITO, TOKUHIRO KIMURA, MICHIHIKO ARAMAKI, KOSUKE IZUMI, YASUNORI OKADA, HIDEKAZU SUZUKI, \\ TAKAO TAKAHASHI, AND KENJIRO KOSAKI \\ Departments of Pediatrics [Y.N., M.A., K.I., T.T., K.K.], Pathology [T.K., Y.O.] and Internal Medicine [H.S.], Keio University School of \\ Medicine, Tokyo 160-8582, Japan
}

\begin{abstract}
VATER association represents a cluster of Vertebral, Anal, Tracheo-Esophageal, Radial and Renal malformations, and caudal regression syndrome is an entity consisting of a spectrum of congenital anomalies of lower spine and hips associated with genitourinary and lower limb defects. The concurrence of various malformations may be explained by a common defect in blastogenesis, but direct evidence is yet to be accumulated. Here, by the use of autofluorescence and the teratogenic effect of adriamycin, we demonstrated that adriamycin administered to eggs of White Leghorns distributes to the caudal portion of the embryo and foregut epithelium and induces caudal regression and tracheal and pulmonary agenesis. The induction of caudal regression syndrome-like anomaly was developmental stage and dose dependent. Embryos with caudal regression demonstrated tracheoesophageal anomalies, one of the defects included in VATER association. The stages at which anomalies were produced corresponded to that of human embryos between days 22 and 26 (Carnegie stages 10-11). In view of the antitumor activity of adriamycin by intercalating to double-stranded DNA of undifferentiated cells undergoing rapid cell division, it is possible that adriamycin had preferentially attacked cells in the caudal end where pronounced proliferation takes place during this narrow period of greatest susceptibility. (Pediatr Res 65: 607-612, 2009)
\end{abstract}

$\mathrm{V}$ ATER association consists of a nonrandom combination of three or more of vertebral $(\mathrm{V})$, anorectal $(\mathrm{A})$, tracheoesophageal (TE), radial and renal (R) congenital defects (1). A number of other defects were reported to occur in addition to the originally described set, including cardiac and genital anomalies, single umbilical artery, limb anomalies other than radial, and caudal regression syndrome (2-7). Based on case series and epidemiologic studies, it has been postulated that defects included in the VATER association result from blastogenetic abnormalities by way of a common pathogenesis

Received September 9, 2008; accepted December 30, 2008.

Correspondence: Kenjiro Kosaki, M.D., Department of Pediatrics, Keio University School of Medicine, 35 Shinanomachi, Shinjuku-ku, Tokyo 160-8582, Japan; e-mail: kkosaki@sc.itc.keio.ac.jp

Supported by the Japanese Ministry of Education, Culture, Sports, Science and Technology; in part by a Grant-in-Aid for Exploratory Research from the Japanese Ministry of Education, Culture, Sports, Science and Technology; and in part by a research grant from the Center of Excellence (COE) program "Basic Study and Clinical Application of the Human Stem Cell Biology and Immunology" (to Y.N.).
$(8-12)$. The combination of anomalies appear to result from genetic and/or environmental "hits" involving several vulnerable primordia at the same time $(4,11)$. The disruption of morphogenetic events at the caudal eminence, an ectoderm covered mass of pluripotent mesenchyme (13), is believed to give rise to malformations including caudal dysgenesis (14), and the failure of the tracheal bud to develop normally from the primitive foregut is considered to be the key event that leads to tracheoesophageal anomalies (15). The concurrence of various malformations may be explained by the "hit" in these areas during blastogenesis, but direct evidence is yet to be accumulated.

A teratogenic model of VATER association has been reported by Thompson et al. (16) who documented the occurrence of esophageal atresia (EA) with tracheoesophageal fistula (TEF) and other anomalies after injecting adriamycin into timed-pregnant rats on days 6-9 of gestation. Adriamycin is an anthracycline, antineoplastic drug. Its proposed mechanism of action includes inhibition of nucleic acid synthesis through intercalation with DNA (17), and it has been widely used in the treatment of many malignancies, including sarcomas, lymphomas, neuroblastoma, and breast cancer (18). There have been several shortcomings in the rat model. First, tissue distribution of adriamycin after administration to dam rat have never been delineated. Second, the actual time point of teratogenic action of adriamycin was obscure because widespread dose given intraperitoneally resulted in variable absorption between the embryos.

To overcome the above inconvenience of adriamycin rat model, Mortell et al. (19) have tried to evaluate teratogenicity of adriamycin by administrating the chemical into the air sac or albumen of chick embryos. Although they could not produce VATER-like anomalies within chick embryos, they have proposed the importance and necessity of a more exact method of gestational staging to identify the initial insult which brings about the multiple anomalies seen in VATER association. By injecting adriamycin into the yolk, rather than

Abbreviation: HH, Hamburger-Hamilton 
into the air sac or albumen, we were able to obtain a consistent pattern of malformation. In this study, we aimed to develop a chick embryonic model of blastogenetic defect by administrating adriamycin at defined developmental stages and by tracing adriamycin in the embryonic tissue with the use of autofluorescence of the drug.

\section{MATERIALS AND METHODS}

Embryos and adriamycin treatment. A stock solution of doxorubicin hydrochloride (Adriamycin; Sigma Chemical, St. Louis, MO) was prepared by dissolving this compound in sterile distilled water at a concentration of 1 $\mathrm{mg} / \mathrm{mL}$. Fertile White Leghorns eggs obtained from a local hatchery were incubated in a forced-draft incubator at $38^{\circ} \mathrm{C}$ with a relative humidity of $70 \%$. The first day of incubation was termed Day 0 . Embryos were incubated for 2 days and developed to stages 10-13 according to the Hamburger-Hamilton (HH) system (20). Doses of $1-5 \mu \mathrm{g}$ of adriamycin in $200 \mu \mathrm{L}$ of sterile distilled water were injected into the yolk using a $1 \mathrm{~mL}$ tuberculin syringe (Terumo Medical Corporation, Tokyo, Japan) through a window that was made on the blunt end of the egg. Control eggs were injected with the same volume of phosphate buffered saline (PBS) as those treated with adriamycin. The windows through which the injections were made were sealed with adhesive tape. To identify the distribution of adriamycin within the embryonic tissue, adriamycin-treated embryos were examined every $3 \mathrm{~h}$ up to $12 \mathrm{~h}$ of incubation following drug injection. For studies of adriamycin-induced pathogenesis, embryos were examined at 24 or 48 additional hours of incubation. The study protocols were approved by the Animal Care committee of Keio University School of Medicine.

Adriamycin imaging by fluorescence. Intraembryonic distribution of adriamycin was observed under LED lights. Lights from 12 blue LEDs (peak wave length, $475 \mathrm{~nm}$ ) were intensified using a concave lens and were projected onto the embryonic specimen through a band-pass filter (BI0060; Asahi Spectra). Emissions from adriamycin fluorescence were observed through a long-pass filter (FF01-520/35-25; bright line). A subset of embryos were fixed in $4 \%$ paraformaldehyde (PFA) and embedded in OCT compound (Tissue-Tek; Sakura Finetek, Torrance, CA). Serial frozen sections $(6-\mu \mathrm{m}$ thick) were cut and mounted on glass slides. Fluorescence of adriamycin was observed with a laser scanning confocal microscope (FLUOVIEW FV300; Olympus). A green $\mathrm{He}-\mathrm{Ne}$ laser line $(543 \mathrm{~nm})$ was used for excitation, and a long pass filter (BA565IF) was used for detection of the emitted light (21). The results were shown with differential interference images of the same sections. Nuclei were stained with a fluorescent nuclear dye TO-PRO-3 (dilution 1:1250; Molecular Probes, Eugene, OR).

Immunohistochemistry. Adriamycin-treated and control embryos were fixed in $4 \%$ PFA and embedded in paraffin. Serial sections (10- $\mu \mathrm{m}$ thick) were cut and placed on glass slides. Apoptotic cells were labeled by immunohistochemistry for single stranded DNA (ssDNA) (22). Correlation between labeling by this method and that by terminal deoxynucleotidyl transferasemediated dUTP-biotin nick end-labeling (TUNEL) method was reported previously (23). Deparaffinized sections were treated with $0.3 \%$ hydrogen peroxide to block endogenous peroxidase activity. They were treated with $0.04 \mathrm{mg} / \mathrm{mL}$ proteinase $\mathrm{K}$ for $5 \mathrm{~min}$ at room temperature and were incubated with rabbit polyclonal anti-single stranded DNA antibody (dilution 1:1000; Dako, Glostrup, Denmark). Sections were then treated with peroxidaseconjugated secondary antibody (ImmPRESS, Vector Laboratories, Burlingame, CA), and the color was developed with diaminobenzidine. Counterstain was done with hematoxylin.

Selected specimens were examined under a dissecting microscope and photographed. A subset of embryos was fixed in $4 \%$ paraformaldehyde and embedded in paraffin, sectioned at $10 \mu \mathrm{m}$, and stained with hematoxylin for subsequent histologic analyses.

BrdU labeling and embryonic culture. After $36 \mathrm{~h}$ in an incubator, $\mathrm{HH}$ stages 10-11 embryos were collected using filter paper disks and were transferred to agar culture plates, as described by Sundin and Eichele (24). Briefly, after partial removal of the thick albumen over the embryo, a dry ring of filter paper was laid on top of the vitelline membrane, and the membrane was cut along the circumference of the filter ring. The ring-embryo-vitelline membrane composite was transferred into warmed Hank's solution to remove yolk and then transferred to agar culture plates, and the embryos were incubated with $100 \mu \mathrm{L}$ of $50 \mu \mathrm{M}$ bromodeoxyuridine (BrdU) (Sigma Chemical) in PBS for $2 \mathrm{~h}$. To visualize BrdU incorporation, 4\% PFA-fixed, paraffin-embedded sections of the embryos were treated with $0.1 \%$ trypsin (Sigma Chemical) at $37^{\circ} \mathrm{C}$ for $10 \mathrm{~min}$ and then $2 \mathrm{~N} \mathrm{HCl}$ at $37^{\circ} \mathrm{C}$ for $30 \mathrm{~min}$. The sections were immunostained using anti-BrdU antibody (clone Bu20a, dilution 1:200, Dako) and peroxidase-conjugated secondary antibody. Diaminobenzidine was used as a chromogen. Counterstain was done with hematoxylin.

\section{RESULTS}

Spatial distribution of adriamycin. Overall distribution of adriamycin in the whole mount embryo was visualized using a stereomicroscope with fluorescence lighting and appropriate filters (Fig. 1). The distribution of adriamycin, as indicated by red signals, was concentrated at the caudal end of the embryo. The maximum signal intensity was observed in the caudal part of the embryo $6 \mathrm{~h}$ after adriamycin administration. Tissue distribution of adriamycin within sectioned embryo was visualized using confocal microscope. Serial sections of HH stage 12 embryos revealed that adriamycin signals were most intense at the caudal end of the embryo and was also present in the foregut epithelium (Figs. 2 and 3). No signal could be detected in control embryos, indicating that fluorescence came only from adriamycin. Co-staining the caudal end section with TO-PRO-3 nuclear dye indicated that signals from adriamycin overlapped with those from TO-PRO-3. Hence, adriamycin was likely to be in the nucleus.

Caudal regression. Caudal regression was demonstrated $48 \mathrm{~h}$ following $5 \mu \mathrm{g}$ of adriamycin injection at $\mathrm{HH}$ stages 10-11 (Fig. 4). Midline structures caudal to the wingbud were absent including the sacral and coccygeal (tail) structures. The embryos were devoid of allantois, an extra-embryonic membrane of birds arising as a pouch or sac, from the hindgut. In birds, it expands greatly between two other membranes, the amnion and chorion, to serve as a temporary respiratory organ while its cavity stores fetal excretions. Adriamycin-treated embryos without allantois did not survive after Day 6. The comparison of the viability and the incidence of caudal regression according to adriamycin injection at four developmental stage groups are given in Table 1. Embryos with absence of the midline structures caudal to the wingbud, the severe type (Fig. 4A), and embryos with tail-like structures, the mild type, were counted as having caudal regression. The presence of caudal regression was evaluated $48 \mathrm{~h}$ following drug administration in each group. Embryos treated before Hamburger-Hamilton (HH) stage 10 had poor survival rate

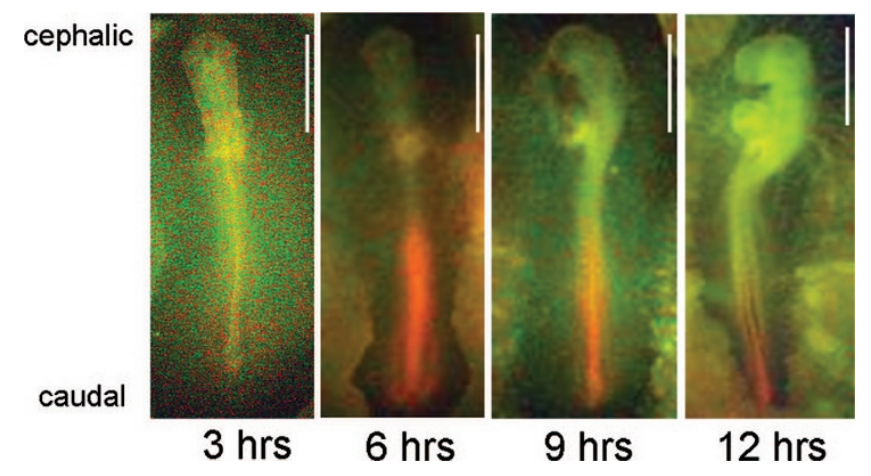

Figure 1. Overall distribution of Adriamycin in the whole mount embryo. Adriamycin was administered to the embryo at $\mathrm{HH}$ stage 11 . The distribution of the adriamycin, as indicated by red signals, was concentrated at the caudal end of the embryo. Maximum signal intensity was observed in caudal portion of the embryo $6 \mathrm{~h}$ after adriamycin administration. Scale bars: $1 \mathrm{~mm}$. 

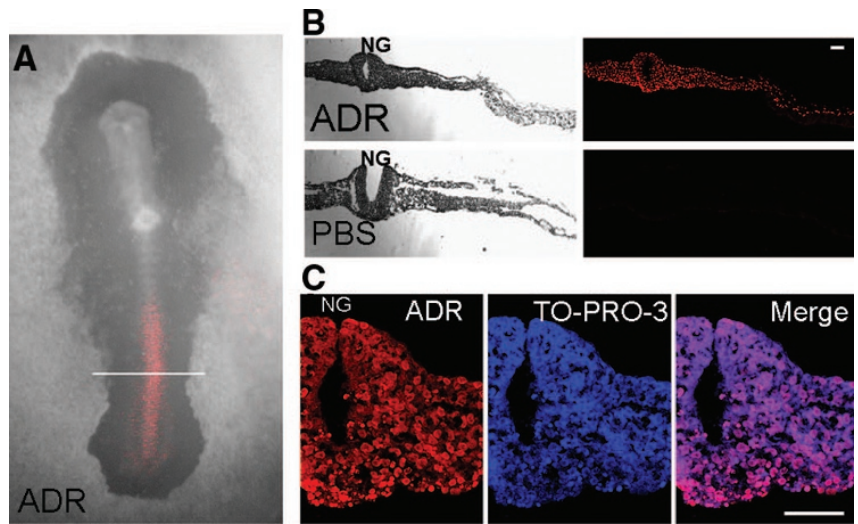

Figure 2. Tissue distribution of adriamycin within the sectioned embryo at stage 12. (A) Signals from adriamycin were demonstrated as superimposed images of the fluorescent and bright field images of the adriamycin-treated embryo. (B) Confocal microscopy images (right) and differential interference images (left) of the transverse sections at the level indicated by the white line in A. Adriamycin fluorescence was detected in cells of the adriamycin-treated embryo (ADR), whereas no signal was observed in the control embryo (PBS). (C) Staining with TO-PRO-3 nuclear dye revealed that signals from adriamycin overlapped with those from TO-PRO-3. NG, neural groove. Scale bars in $B$ and $C, 50 \mu \mathrm{m}$
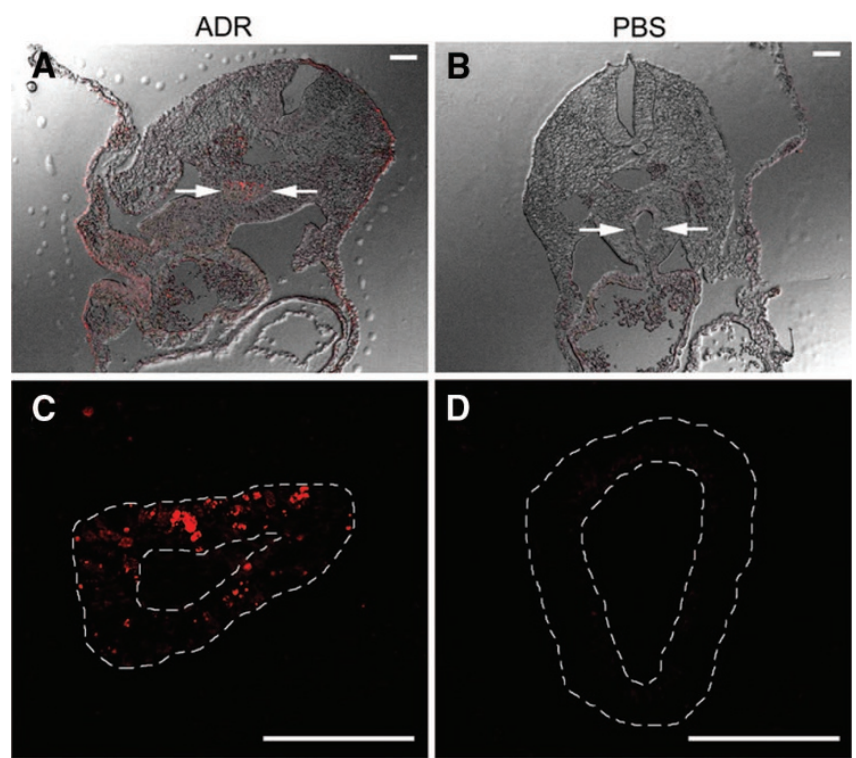

Figure 3. Confocal microscopy images of embryos $24 \mathrm{~h}$ after adriamycin $(A$ and $C$ ) or PBS ( $B$ and $D$ ) administration at HH stage 11. $(A, B)$ Superimposed images of adriamycin fluorescence and differential interference. Arrows indicate foregut. $(C, D)$ Magnified views of the foregut (adriamycin fluorescence signal only). Foregut epithelia are outlined by white dotted lines. Fluorescent signals from adriamycin were detected in the foregut epithelial cells of the adriamycin-treated embryo $(A, C)$. Scale bars: $100 \mu \mathrm{m}$.

including control embryos. Administration of $2-5 \mu \mathrm{g}$ of adriamycin at $\mathrm{HH}$ stages 10 and 11 led to caudal regression in more than $90 \%$ of the adriamycin-treated embryos, whereas less than $20 \%$ of $1 \mu \mathrm{g}$ administered embryos developed this anomaly. The incidence of caudal regression decreased as embryonic stages at the time of treatment progressed. When 2 $\mu \mathrm{g}$ of adriamycin was given at HH stages $14-16$, only $10 \%$ of treated embryos developed caudal regression. The severity of caudal regression was also influenced by the stage at which the drug was injected and by the dosage of the drug. All
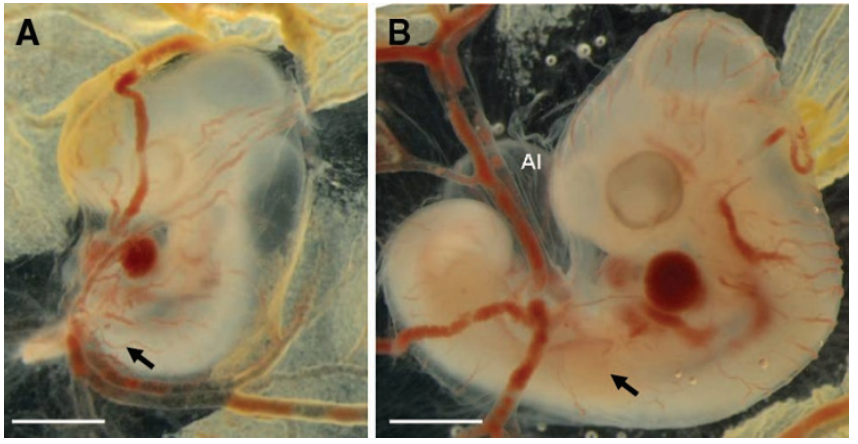

Figure 4. Overview images of adriamycin-treated and control embryos. $(A)$ Forty-eight hours following $5 \mu \mathrm{g}$ of adriamycin injection and (B) PBS injection at $\mathrm{HH}$ stage 10 , respectively. Midline structures caudal to the wingbud (arrows) were lacking in the adriamycin treated embryo. Al, allantois. Scale bars: $1 \mathrm{~mm}$.

Table 1. Incidence of caudal regression after adriamycin injection at various dosages and developmental stages

\begin{tabular}{|c|c|c|c|c|}
\hline & \multicolumn{2}{|c|}{ Viable embryo } & \multicolumn{2}{|c|}{ Caudal regression } \\
\hline & $n$ & $\%$ & $n$ & $\%$ \\
\hline \multicolumn{5}{|l|}{ НH 7-9 } \\
\hline Control ADR & $7 / 20$ & $35.0 \%$ & 0 & 0 \\
\hline $1 \mu \mathrm{g}$ & $5 / 24$ & $20.8 \%$ & $4 / 5$ & $80.0 \%$ \\
\hline $2 \mu \mathrm{g}$ & $1 / 24$ & $4.2 \%$ & $1 / 1$ & $100 \%$ \\
\hline $5 \mu \mathrm{g}$ & $1 / 26$ & $3.8 \%$ & $1 / 1$ & $100 \%$ \\
\hline \multicolumn{5}{|l|}{ HН $10-11$} \\
\hline Control ADR & $22 / 24$ & $91.7 \%$ & 0 & 0 \\
\hline $1 \mu \mathrm{g}$ & $21 / 24$ & $87.5 \%$ & $4(3)^{*} / 21$ & $19.0 \%$ \\
\hline $2 \mu \mathrm{g}$ & $24 / 32$ & $75.0 \%$ & $22 / 24$ & $91.7 \%$ \\
\hline $5 \mu g$ & $40 / 54$ & $74.0 \%$ & $38 / 40$ & $95.0 \%$ \\
\hline \multicolumn{5}{|l|}{ HH12-13 } \\
\hline Control & $19 / 20$ & $95.0 \%$ & 0 & 0 \\
\hline $1 \mu \mathrm{g}$ & $28 / 34$ & $82.3 \%$ & 0 & 0 \\
\hline $2 \mu \mathrm{g}$ & $37 / 42$ & $88.1 \%$ & $24(5) * / 37$ & $64.9 \%$ \\
\hline $5 \mu \mathrm{g}$ & $35 / 41$ & $85.4 \%$ & $24(6)^{*} / 35$ & $68.6 \%$ \\
\hline \multicolumn{5}{|l|}{ НH14-16 } \\
\hline Control ADR & $17 / 18$ & $94.4 \%$ & 0 & 0 \\
\hline $1 \mu \mathrm{g}$ & $19 / 20$ & $95.0 \%$ & 0 & 0 \\
\hline $2 \mu \mathrm{g}$ & $20 / 25$ & $80.0 \%$ & $2(1)^{*} / 20$ & $10.0 \%$ \\
\hline $5 \mu \mathrm{g}$ & $28 / 35$ & $80.0 \%$ & $8(2)^{*} / 28$ & $28.6 \%$ \\
\hline
\end{tabular}

$*(n)$ indicates number of embryos with tail-like structures.

embryos treated with 2 and $5 \mu \mathrm{g}$ of adriamycin at $\mathrm{HH}$ stages 10-11 presented with severe type of caudal regression, whereas mild type was found within embryos treated at later stages or given lower dosages (Table 1). These results revealed that caudal regression developed in a dose related manner and that the period of greatest susceptibility for this malformation was between HH stages 10-13 with HH stages 10-11 being most sensitive.

Comparison of transverse histologic sections at the level below the wingbud in adriamycin-treated and control embryos at $\mathrm{HH}$ stage 17 demonstrated excessive cell death in the adriamycin-treated embryos, as assessed by anti-single stranded DNA immunostaining (Fig. 5). Cell death of 24-h adriamycin-treated embryo was evident in cells caudal to the level of wingbud, with many positively stained cells observed in the neural tube, sclerotome, and somites (Fig. 5). The caliber of the caudal end of the neural tube was smaller and 


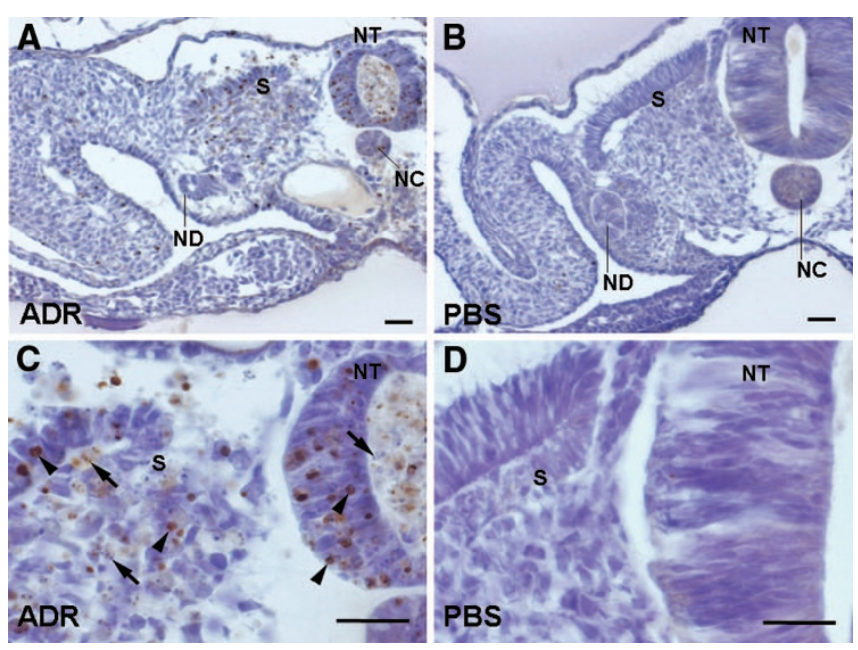

Figure 5. Comparison of transverse histologic sections at the level below wingbud in adriamycin-treated $(A$ and $C$ ) and control $(B$ and $D)$ embryos. $C$ and $D$ are magnified views of $A$ and $B$, respectively. Adriamycin or PBS was administered to embryos at $\mathrm{HH}$ stage 11, and the embryos were fixed $24 \mathrm{~h}$ later. Sections were immunostained with anti-single stranded DNA antibody and counterstained with hematoxylin. In the adriamycin-treated embryo ( $A$ and $C$ ) many immunoreactive cells (nuclei) were observed in the neural tube and somite (arrowheads in $C$ ), and nuclear debris was also present (arrows in $C$ ). In the control embryo $(B$ and $D)$, only a small number of immunoreactive cells were found in the lateral plate mesoderm, and no nuclear debris was seen. NT, neural tube; S, somite (dermomyotome and sclerotome); NC, notochord; ND, nephric duct. Scale bars, $25 \mu \mathrm{m}$.

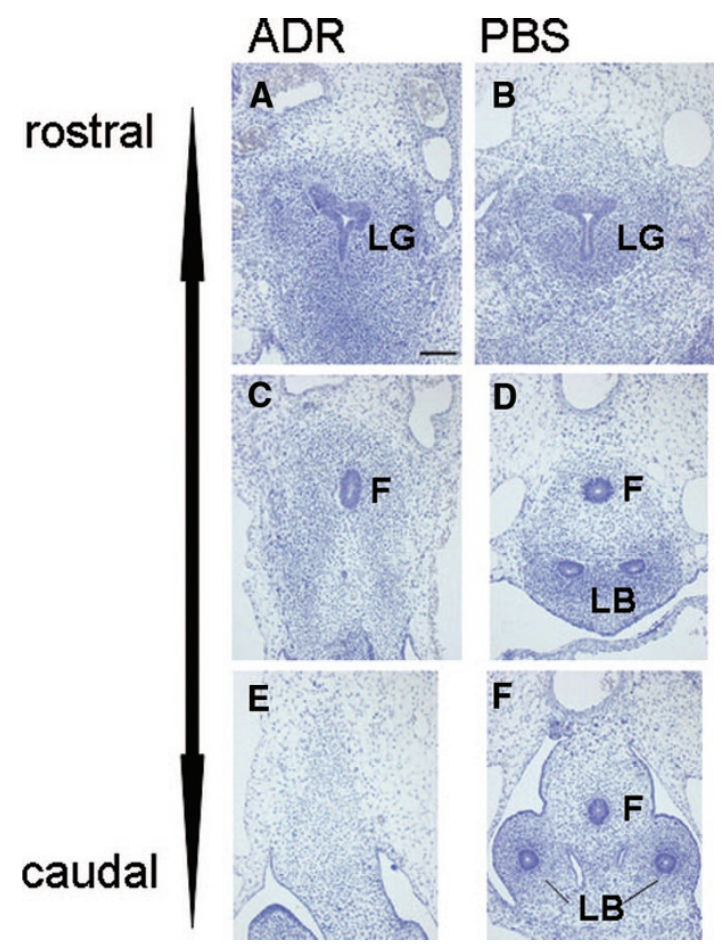

Figure 6. Rostral to caudal comparison of transverse histologic sections of adriamycin-treated and control embryos. $(A, C, E)$ Seventy-two hours post adriamycin-treated embryos and $(B, D, F)$ control embryos at levels of laryngotracheal groove $(A, B)$, lung buds $(C, D)$, and lower esophagus $(E, F)$. In the adriamycin-treated embryo, the caliber of upper foregut segment narrowed down as it developed caudally and eventually disappeared within the mesenchyme. LG, laryngotracheal groove; F, foregut; LB, lungbud. Scale bar, $100 \mu \mathrm{m}$. many of the somite tissues were degenerated. The structure of the notochord was relatively spared.

Vascular anomalies, especially of the dorsal aortae, were carefully studied on histologic sections. The dorsal aortae of the treated embryos were not enlarged compared with those of the control. Furthermore, there were no breaks in the walls of dorsal aortae which led to formation of caudal hematoma as described by Kaplan and Grabowski (25).

Foregut atresia and tracheal and pulmonary agenesis. Of the 12 histologically assessed embryos given $5 \mu \mathrm{g}$ of adriamycin at $\mathrm{HH}$ stages 10-11, eight demonstrated foregut atresia with tracheal and pulmonary agenesis (Fig. 6), one had foregut stenosis with tracheal and pulmonary agenesis, one had foregut stenosis, and two had normal foregut and pulmonary development. All of the control embryos $(n=6)$ had normal foregut development and tracheal separation was completed within $48 \mathrm{~h}$ following PBS injection. None of the adriamycintreated embryos presented with tracheoesophageal fistula. Regarding other anomalies, a dorsally swollen neural tube was found in three of the adriamycin-treated embryos.

BrdU labeling. Relative increase in number of BrdU incorporated cells was observed in the caudal sections compared with the rostral sections (Fig. 7). Foregut epithelia did not present with significant difference in BrdU incorporation with other neighboring tissues.

\section{DISCUSSION}

Here, we have demonstrated that exposure of chick embryo to adriamycin leads to caudal regression and complete lack of respiratory system with foregut atresia. These results were in line with previous rat studies in which malformation of the foregut and caudal eminence derived organs, including esophageal and intestinal atresia, tracheoesophageal fistula, renal and anorectal anomalies, were induced by embryonic exposure to adriamycin $(16,26,27)$. This study not only replicated embryonic toxicity of adriamycin in a species other than rodents, but also strengthened conclusion of previous studies in three aspects. First, our system allowed us to document tissue distribution of adriamycin within the embryo by exploiting the autofluorescence of the drug. In previously described rat models, tissue distribution of adriamycin after administration to dam rat have never been delineated. Second, unlike the rat model in which adriamycin was administered intraperitoneally to pregnant rats, we were able to administer the teratogen directly at a defined developmental stage. This allowed us to identify the actual time point of the teratogenic effect on the embryo. Third, a more accurate dose response relationship between the teratogen and development of defects could be analyzed in the chick embryonic model. The dosage of drug per embryo was precisely controlled in our model whereas in the rat model the actual amount of adriamycin that reached to the target embryonic tissue could have been variable because adriamycin delivery was dependent on peritoneal absorption in the dam and transplacental transfer.

Both caudal regression and tracheoesophageal anomaly constitute prototypic defects of VATER association which has traditionally been classified as "blastogenesis defect" based on 


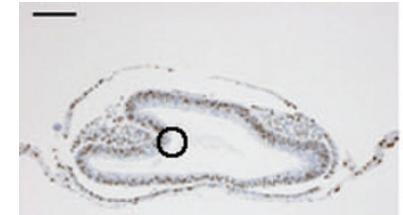

A

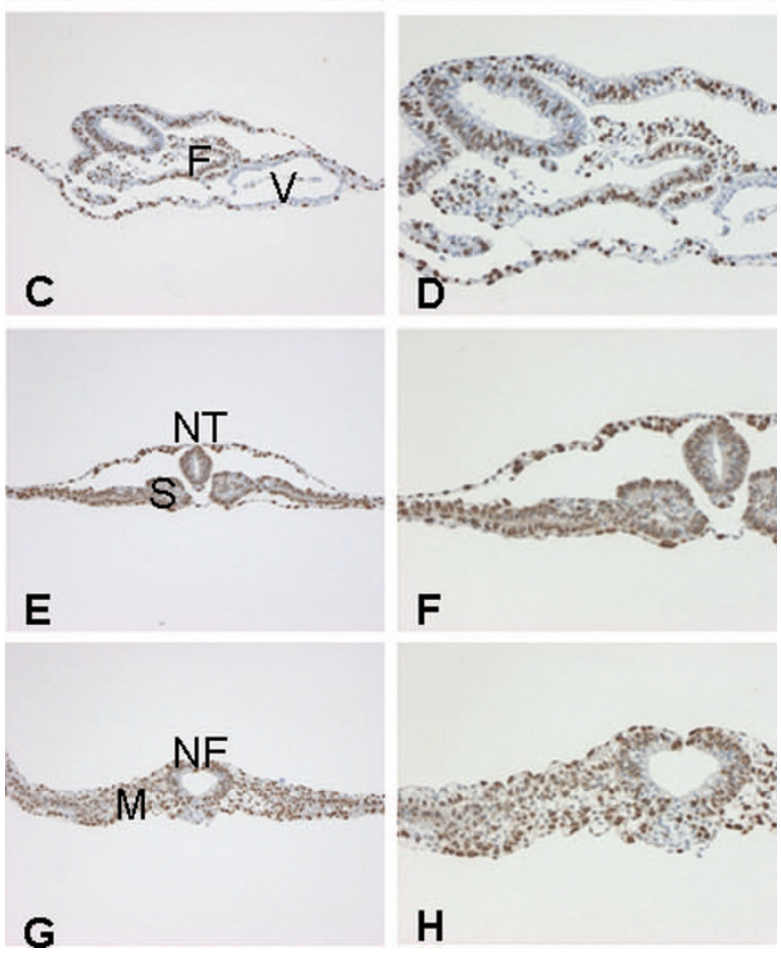

Figure 7. Comparison of BrdU incorporation in rostral and caudal parts of the embryo. Sections of a HH stage 11 embryo at the levels of optic cup $(A$, $B)$, vitelline vein $(C, D)$, first somite $(E, F)$, and neural fold $(G, H)$ are shown. $B, D, F, H$ are magnified views of $A, C, E, G$, respectively. Relative increase in the number of BrdU incorporated cells (brown) was found in the caudal sections $(E-H)$, especially in the somite, mesoderm, and the neural tube. There was no significant difference in BrdU staining in the foregut epithelia compared with other neighboring tissues. O, optic vesicle; $\mathrm{V}$, vitelline vein; F, foregut; S, somite; NT, neural tube; NF, neural fold; M, mesoderm. Scale bar in A, $100 \mu \mathrm{m}$; Scale bar in B, $50 \mu \mathrm{m}$.

a deliberate embryological inference (11). Here, the term blastogenesis refers to a rather broad period that includes all stages of development within the first 4 wk of human development (days 1-28, Carnegie stages 1-12). Our model enabled us to clarify that $\mathrm{HH}$ stages $10-13$ represent the susceptible period as far as caudal regression is concerned. More specifically, the severe type of caudal regression was concentrated in embryos treated at earlier stages within this period (HH stages 10-11). This period corresponds to embryonic development between days 22 and 26 (Carnegie stages 10-11) in humans. Precise definition of the vulnerable period, rather than "blastogenesis period," would be helpful when inferring the pathogenesis of diabetic embryopathy, major feature of which include caudal regression.

The fluorescence data suggested that adriamycin was concentrated in multiple cell types (neural tube, somites, paraxial mesoderom) of the caudal embryo and selectively in foregut epithelium. The caudal distribution of adriamycin included the caudal eminence, an ectoderm-covered mass of pluripotent mesenchyme (13). It is the growth center of the caudal end of the embryo and provides mesenchyme for the formation of the notochord, somites (including caudal vertebrae), hindgut, neural tube, hind limbs, and blood vessels $(13,28)$. The basis for such selective distribution of the drug seems to be a key to its teratogenic effects. Considering that adriamycin exerts its antitumor activity by intercalating to double-stranded DNA of undifferentiated cells undergoing rapid cell division (29), it is possible that adriamycin had preferentially attacked cells in tissues where pronounced proliferation takes place at the time of drug administration. To identify the areas of active proliferation, we examined BrdU incorporation within the cells of the developing embryo. BrdU is a thymidine analog which incorporates into newly synthesized DNA strands of actively proliferating cells (30). Relatively large number of BrdU incorporated cells was seen in the caudal sections compared with the rostral sections. Furthermore, Mac Auley et al. have determined the duration of the cell cycle for mesodermal and ectodermal cells of rat embryos during gastrulation and found that the primitive streak, the former region of caudal eminence (31), was the region of highest proliferative activity (32). Taken together, one of the possible mechanisms of adriamycin distribution to the caudal end may reflect the high rates of cell proliferation. On the other hand, we did not find significant difference in BrdU incorporation in the foregut epithelium with other neighboring tissues. The underlying mechanism(s) of selective distribution of adriamycin within foregut epithelium is yet to be discovered.

In the chick embryonic model, caudal regression was the obligate defect in all the affected embryos. In view of the notion that disruption of the morphogenetic events at the caudal eminence would give rise to malformations including caudal dysgenesis (14), the observation that maximal signal intensity of adriamycin was present in caudal portion of the embryo may account for the vulnerability of the caudal structure. The pathogenetic mechanism by which disruption in caudal eminence leads to caudal regression has not been delineated. In the literature, the vascular theory has been invoked by some authors to account for the pathogenesis of caudal regression. Kaplan et al. (25) treated early chick embryos with trypan blue and obtained caudal regression. Embryos treated with trypan blue exhibited a high incidence of caudal hematoma, which led to caudal regression at later developmental stages. The hisotological study of these embryos revealed following sequential changes: 1) enlargement of the dorsal aortae, 2) rupture of the aortae and bleeding into tissue spaces, 3) structural damage due to the expanding hematoma, 4) necrosis, 5) resorption of dead tissues, and 6) caudal regression. In our adriamycin-treated embryos, massive cell death of the somites, sclerotome and neural tube at the level below the wingbud (processes 4-6) were observed as well. However, we did not find any of the above-mentioned vascular changes (processes 1-3) within the vascular system in $\mathrm{HH}$ stages 10-13 treated embryos. Hence, it is unlikely that caudal regression was mediated by vascular disruption at least in the present chick model. 
Caudal regression syndrome-like anomalies has been reported to develop in glucose-treated and insulin-treated chick embryos $(33,34)$. In both studies, the mortality and morbidity were developmental stage dependent. The vulnerable period defined in these studies were rather broad (i.e. between days 0 and 2) (33), but included HH stages 10-11, the period of greatest susceptibility for adriamycin teratogenicity for caudal regression. How inhibition of mitogenesis by adriamycin and disruption of glucose metabolism by addition of glucose or by insulin lead to the same phenotypic outcome, caudal regression, is yet to be clarified.

In humans, no cases with VATER association or caudal regression syndrome have been identified in the retrospective review on fetal outcomes following chemotherapy with an anthracycline-containing regimen in pregnant cancer patients (35). The discrepancy between human and chick may be caused by species specificity or lack of human cases with adriamycin administration within the vulnerable period.

In summary, adriamycin injected to HH stages 10-11 chick embryos accumulated in the caudal portion of the embryo and foregut epithelium and induced caudal regression-like anomalies and tracheoesophageal anomalies. Our study revealed that there might be multiple mechanisms in which adriamycin exerts its teratogenic effects in growing embryo. One of the possible mechanism(s) may include the disruption of the cell cycle of rapidly proliferating cells within the caudal eminence during this narrow period. Further study is needed to identify the underlying mechanism(s) of selective distribution of adriamycin within the foregut epithelia.

Acknowledgments. We thank Yuko Hashimoto for technical support.

\section{REFERENCES}

1. Quan L, Smith DW 1973 The VATER association. Vertebral defects, Anal atresia, T-E fistula with esophageal atresia, Radial and Renal dysplasia: a spectrum of associated defects. J Pediatr 82:104-107

2. Kallen B 1987 Caudal aplasia. In: Myrianthopoulos NC (ed) Handbook of Clinical Neurology: Malformations. Elsevier Science, Amsterdam, pp 509-518

3. Duncan PA, Shapiro LR 1993 Interrelationships of the hemifacial microsomiaVATER, VATER, and sirenomelia phenotypes. Am J Med Genet 47:75-84

4. Lubinsky M 1986 VATER and other associations: historical perspectives and modern interpretations. Am J Med Genet Suppl 2:9-16

5. Jones K 2005 Smith's Recognizable Patterns of Human Malformation, 6th Ed. Elsevier Saunders, Philadelphia, pp 756-759

6. Schuler L, Salzano FM 1994 Patterns in multimalformed babies and the question of the relationship between sirenomelia and VACTERL. Am J Med Genet 49:29-35

7. Temtamy SA, Miller JD 1974 Extending the scope of the VATER association: definition of the VATER syndrome. J Pediatr 85:345-349

8. Botto LD, Khoury MJ, Mastroiacovo P, Castilla EE, Moore CA, Skjaerven R, Mutchinick OM, Borman B, Cocchi G, Czeizel AE, Goujard J, Irgens LM, Lancaster
PA, Martinez-Frias ML, Merlob P, Ruusinen A, Stoll C, Sumiyoshi Y 1997 The spectrum of congenital anomalies of the VATER association: an international study. Am J Med Genet 71:8-15

9. Kallen B, Winberg J 1974 Caudal mesoderm pattern of anomalies: from renal agenesis to sirenomelia. Teratology 9:99-111

10. Russell LJ, Weaver DD, Bull MJ 1981 The axial mesodermal dysplasia spectrum. Pediatrics 67:176-182

11. Opitz JM 1993 Blastogenesis and the primary field in human development. Birth Defects Orig Artic Ser 29:3-37

12. Martinez-Frias ML, Frias JL, Opitz JM 1998 Errors of morphogenesis and developmental field theory. Am J Med Genet 76:291-296

13. Muller F, O'Rahilly R 2004 The primitive streak, the caudal eminence and related structures in staged human embryos. Cells Tissues Organs 177:2-20

14. O’Rahilly R, Muller F 1989 Interpretation of some median anomalies as illustrated by cyclopia and symmelia. Teratology 40:409-421

15. Merei JM, Farmer P, Hasthorpe S, Qi BQ, Beasley SW, Myers NA, Hutson JM 1997 Timing and embryology of esophageal atresia and tracheo-esophageal fistula. Anat Rec 249:240-248

16. Thompson DJ, Molello JA, Strebing RJ, Dyke IL 1978 Teratogenicity of adriamycin and daunomycin in the rat and rabbit. Teratology 17:151-157

17. Marco A, Arcamone F 1975 DNA complexing antibiotics: daunomycin, adriamycin and their derivatives. Arzneimittelforschung 25:368-374

18. Young RC, Ozols RF, Myers CE 1981 The anthracycline antineoplastic drugs. N Engl J Med 305:139-153

19. Mortell A, Giles J, Bannigan J, Puri P 2003 Adriamycin effects on the chick embryo. Pediatr Surg Int 19:359-364

20. Hamburger V, Hamilton HL 1951 A series of normal stages in the development of the chick embryo. J Morphol 88:49-92

21. Primeau AJ, Rendon A, Hedley D, Lilge L, Tannock IF 2005 The distribution of the anticancer drug Doxorubicin in relation to blood vessels in solid tumors. Clin Cancer Res 11:8782-8788

22. Maeda M, Sugiyama T, Akai F, Jikihara I, Hayashi Y, Takagi H 1998 Single stranded DNA as an immunocytochemical marker for apoptotic change of ischemia in the gerbil hippocampus. Neurosci Lett 240:69-72

23. Watanabe I, Toyoda M, Okuda J, Tenjo T, Tanaka K, Yamamoto T, Kawasaki H, Sugiyama T, Kawarada Y, Tanigawa N 1999 Detection of apoptotic cells in human colorectal cancer by two different in situ methods: antibody against single-stranded DNA and terminal deoxynucleotidyl transferase-mediated dUTP-biotin nick endlabeling (TUNEL) methods. Jpn J Cancer Res 90:188-193

24. Sundin O, Eichele G 1992 An early marker of axial pattern in the chick embryo and its respecification by retinoic acid. Development 114:841-852

25. Kaplan S, Grabowski CT 1967 Analysis of trypan blue-induced rumplessness in chick embryos. J Exp Zool 165:325-335

26. Diez-Pardo JA, Baoquan Q, Navarro C, Tovar JA 1996 A new rodent experimental model of esophageal atresia and tracheoesophageal fistula: preliminary report. J Pediatr Surg 31:498-502

27. Merei J, Hasthorpe S, Farmer P, Hutson JM 1999 Visceral anomalies in prenatally adriamycin-exposed rat fetuses: a model for the VATER association. Pediatr Surg Int 15:11-16

28. Muller F, O'Rahilly R 1983 The first appearance of the major divisions of the human brain at stage 9. Anat Embryol (Berl) 168:419-432

29. Kiyomiya K, Matsuo S, Kurebe M 2001 Differences in intracellular sites of action of Adriamycin in neoplastic and normal differentiated cells. Cancer Chemother Pharmacol 47:51-56

30. Hardonk MJ, Harms G 1990 The use of 5'-bromodeoxyuridine in the study of cell proliferation. Acta Histochem Suppl 39:99-108

31. O'Rahilly R, Muller F 1987 Developmental stages in human embryos: including a revision of streeter's 'Horizon' and a survey of the Carnegie Collection. Carnegie Institution of Washington, Washington, DC, pp 637

32. Mac Auley A, Werb Z, Mirkes PE 1993 Characterization of the unusually rapid cell cycles during rat gastrulation. Development 117:873-883

33. Julian D, Abbott UK 1998 An avian model for comparative studies of insulin teratogenicity. Anat Histol Embryol 27:313-321

34. Datar S, Bhonde RR 2005 Shell-less chick embryo culture as an alternative in vitro model to investigate glucose-induced malformations in mammalian embryos. Rev Diabet Stud 2:221-227

35. Germann N, Goffinet F, Goldwasser F 2004 Anthracyclines during pregnancy: embryo-fetal outcome in 160 patients. Ann Oncol 15:146-150 